\title{
New chiral gravity
}

\author{
Luca Ciambelli, ${ }^{*}$ Stéphane Detournay $\oplus^{\dagger}{ }^{\dagger}$ and Antoine Somerhausen $\oplus^{\ddagger}$ \\ Physique Théorique et Mathématique, Université libre de Bruxelles and International Solvay Institutes, \\ Campus Plaine C.P. 231, B-1050 Bruxelles, Belgium
}

(Received 15 August 2020; accepted 21 October 2020; published 16 November 2020)

\begin{abstract}
The phase space of three-dimensional gravity with Compere-Song-Strominger (CSS) boundary conditions is endowed with asymptotic symmetries consisting in the semidirect product of a Virasoro and a $\hat{u}(1)$ KačMoody algebra, and contains Bañados-Teitelboim-Zanelli (BTZ) black holes whose entropy can be accounted for by the degeneracy of states of a warped Conformal Field Theory (CFT). By embedding these boundary conditions in topologically massive gravity, we observe the existence of two special points in the space of couplings parametrized by the $\mathrm{AdS}_{3}$ radius $\ell$ and the Chern-Simons coupling $\mu$. When $\mu= \pm \frac{1}{\ell}$, the asymptotic symmetries reduce to either a chiral Virasoro algebra or a pure $\hat{u}(1)$ Kač-Moody current algebra. At those points, black holes have positive energy while that of linearized excitations are non-negative.
\end{abstract}

DOI: 10.1103/PhysRevD.102.106017

\section{INTRODUCTION}

Lower-dimensional gravity models have appeared over the years as a fertile playground to address fundamental questions in quantum gravity, such as the black hole entropy problem $[1,2]$ of the black hole information paradox $[3,4]$. In particular, Einstein-Hilbert gravity in $(2+1)$ dimensions [5-8] with a negative cosmological constant $\Lambda=-\frac{1}{\ell^{2}}$ has emerged as a very insightful toy model. Even though the theory has no bulk propagating degrees of freedom, it has asymptotically $\mathrm{AdS}_{3}$ (BTZ) black hole solutions $[9,10]$, as well as massless gravitons which can be viewed as propagating on the boundary. Furthermore, the phase space of $\mathrm{AdS}_{3}$ gravity admits a nontrivial action of the 2-dimensional conformal group [11], which appeared as the first hint of a deep connection between a gravity theory in AdS space and a CFT in one dimension less, later unravelled by the AdS/CFT correspondence [12]. Remarkably, the Bekenstein-Hawking entropy of the BTZ black holes could be reproduced by a counting of states in the corresponding dual CFT [13], opening a new perspectives on the black hole entropy problem. More recently, decisive progress has also been achieved regarding the black hole information paradox relying on a new understanding of how to compute the entropy of Hawking

\footnotetext{
*luca.ciambelli@ulb.ac.be

†sdetourn@ulb.ac.be

antoine.somerhausen@ulb.be
}

Published by the American Physical Society under the terms of the Creative Commons Attribution 4.0 International license. Further distribution of this work must maintain attribution to the author(s) and the published article's title, journal citation, and DOI. Funded by SCOAP. radiation $[14,15]$. The key point is the inclusion in the gravitational path integral used to compute the entropy of new saddles arising as complexified wormholes [16], which could be explicitly identified in two-dimensional Jackiw-Teitelboim gravity [17-19] (which can be viewed as the dimensional reduction of $3 \mathrm{~d}$ gravity $[20,21]$ ) coupled to matter.

The BTZ entropy derivation relied only on conformal symmetry and few additional assumptions such as unitarity [22] and sparseness of the spectrum [23]. The precise nature of the $2 \mathrm{~d}$ CFT dual to pure gravity remained therefore elusive until 2007 when Witten, assuming holomorphic factorization, proposed that the theory should be an extremal CFT, establishing intriguing connections between quantum gravity, group and number theory [24]. Later Maloney and Witten computed the $3 \mathrm{~d}$ gravity partition function as a sum over topologies and found that the result could not be interpreted as a trace over some CFT Hilbert space [25]. Moreover, there are arguments (although no proof) that extremal CFTs could not exist for large central charge, i.e., in the semi-classical regime [26-28]. Various steps have been taken toward fixing them [29-32], but it is still unclear whether pure $3 d$ gravity could make sense as a quantum theory. Few months after Witten's proposal, Li, Song and Strominger suggested an alternative for a fully consistent and unitary gravity theory with partition function that of an extremal CFT under the name chiral gravity [33]. By supplementing Einstein gravity with a gravitational Chern-Simons term, they argued that the resulting theory-topologically massive gravity (TMG) $[34,35]$ - becomes chiral at a specific point in coupling space. Heated debates ensued as to determine whether the theory was actually chiral and unitary (see, e.g., [36-55]), leading to unexpected correspondences between critically 
tuned $\mathrm{AdS}_{3}$ gravity and a logarithmic CFT [56-60]. Whether chiral gravity could exist as a unitary truncation of the nonunitary logarithmic CFT that is dual to TMG at the critical point is still an open question, but still stands out as a candidate for the simplest and potentially solvable model including quantum black holes.

The core of this work is based on the recent observations that pure $\mathrm{AdS}_{3}$ ought not to be dual to 2d CFT in the first place. In defining the classical phase space of a physical theory, boundary conditions play as an important role as the action or the equations of motion. It was noticed in a series of works that besides the classic Brown-Henneaux boundary conditions with conformal symmetry, a variety of alternative consistent choices could exist [61-69]. In particular, it was observed by Compere, Song and Strominger (CSS) that pure 3d gravity with appropriate boundary conditions (referred to as CSS) has asymptotic symmetries consisting in the semidirect product of a Virasoro and a $\hat{u}(1)$ Kač-Moody algebra. Those symmetries are those of a new type of $2 \mathrm{~d}$ field theories, Warped CFTs (WCFTs) [70,71]. The study of WCFTs was triggered by the search for holographic duals to the near-horizon region of extremal black holes [72-74] and warped $\mathrm{AdS}_{3}\left(\mathrm{WAdS}_{3}\right)$ spaces [75,76], and they have attracted a lot of attention in recent years [61,77-93]. The main goal of the present work we will be to show that embedding CSS boundary conditions in TMG allows to identify two special points in coupling space where the warped symmetries either reduce to a chiral Virasoro algebra or to a $\hat{u}(1)$ Kač-Moody algebra, thereby possibly providing the simplest example of a gravity theory including black holes.

\section{CSS BOUNDARY CONDITIONS}

The general solution of Einstein equations with CSS boundary conditions is given by [61]

$$
\begin{aligned}
\mathrm{d} s^{2}= & \ell^{2} \frac{\mathrm{d} \rho^{2}}{\rho^{2}}-\rho^{2} \mathrm{~d} x^{+}\left(\mathrm{d} x^{-}-\partial_{+} \bar{P} \mathrm{~d} x^{+}\right) \\
& +4 G \ell\left[\bar{L} \mathrm{~d} x^{+2}+\Delta\left(\mathrm{d} x^{-}-\partial_{+} \bar{P} \mathrm{~d} x^{+}\right)^{2}\right] \\
& -\frac{16 G^{2} \ell^{2}}{\rho^{2}} \Delta \bar{L} \mathrm{~d} x^{+}\left(\mathrm{d} x^{-}-\partial_{+} \bar{P} \mathrm{~d} x^{+}\right),
\end{aligned}
$$

with $\ell$ the AdS radius, $G$ Newton's constant, dimensionless chiral functions $\bar{L}\left(x^{+}\right)$and $\partial_{+} \bar{P}\left(x^{+}\right)$(which is also periodic) and $\Delta$ a constant. The conformal boundary is located at $\rho \rightarrow \infty$ and $x^{ \pm}=\frac{t}{\ell} \pm \phi$ with $\phi \sim \phi+2 \pi$.

BTZ black holes with mass $M$ and angular momentum $J$ are included in this family of metrics for vanishing $\partial_{+} \bar{P}$ and $\bar{L}\left(x^{+}\right)=\bar{\Delta}$, with $\ell M=\Delta+\bar{\Delta}$ and $J=\Delta-\bar{\Delta}$. Global $\mathrm{AdS}_{3}$ is recovered as usual for $M=-1 / 8 G$ and $J=0$.

Infinitesimal transformations leaving (1) invariant are given by asymptotic Killing vectors (AKVs)

$$
\xi=\epsilon \partial_{+}+\left(\sigma+\frac{\ell^{2}}{2 \rho^{2}} \partial_{+}^{2} \epsilon\right) \partial_{-}-\frac{\rho}{2} \partial_{+} \epsilon \partial_{\rho}+\mathcal{O}\left(\ell^{4} / \rho^{4}\right) .
$$

and depend on two chiral arbitrary functions $\epsilon\left(x^{+}\right)$and $\sigma\left(x^{+}\right)$. Expanding in Fourier modes, one finds the conserved charges

$\mathcal{L}_{m}:=Q_{\epsilon=e^{i m x^{+}}}=\frac{1}{2 \pi} \int_{0}^{2 \pi} \mathrm{d} \phi e^{i m x^{+}}\left(\bar{L}-\Delta\left(\partial_{+} \bar{P}\right)^{2}\right)$,
$\mathcal{P}_{m}:=Q_{\sigma=e^{i m x^{+}}}=\frac{1}{2 \pi} \int_{0}^{2 \pi} \mathrm{d} \phi e^{i m x^{+}}\left(\Delta+2 \Delta \partial_{+} \bar{P}\right)$,

satisfying a Virasoro-Kač-Moody algebra:

$$
\begin{aligned}
i\left\{\mathcal{L}_{m}, \mathcal{L}_{n}\right\} & =(m-n) \mathcal{L}_{m+n}+\frac{c_{R}}{12} m^{3} \delta_{m,-n}, \\
i\left\{\mathcal{L}_{m}, \mathcal{P}_{n}\right\} & =-n \mathcal{P}_{m+n}, \\
i\left\{\mathcal{P}_{m}, \mathcal{P}_{n}\right\} & =\frac{k_{K M}}{2} m \delta_{m,-n} .
\end{aligned}
$$

with

$$
c_{R}=\frac{3 \ell}{2 G}, \quad k_{K M}=-4 \Delta=-4 \mathcal{P}_{0} .
$$

Notice the unusual fact that the level is charge-dependent. A WCFT with this symmetry algebra is said to be in quadratic ensemble, and can be brought to canonical form either using state-dependent AKVs or a nonlocal redefinition of the charges $[71,93,94]$. In that case, the level is a negative constant. While this indicates non-unitarity of the theory, it appears to be a feature of holographic WCFTs and does not prevent, for instance, to apply techniques of the modular bootstrap to constrain the spectrum of the theory $[94,95]$.

\section{CSS IN TMG}

Topologically massive gravity [34] is described by the following three-dimensional action

$$
I_{\mathrm{TMG}}=\frac{1}{16 \pi G} \int_{M} \mathrm{~d}^{3} x \sqrt{-g}(R-2 \Lambda)+\frac{1}{\mu} I_{C S} .
$$

The gravitational Chern-Simons term $I_{\mathrm{CS}}$ is given by

$I_{\mathrm{CS}}=\frac{1}{32 \pi G} \int_{M} \mathrm{~d}^{3} x \sqrt{-g} \epsilon^{\lambda \mu \nu} \Gamma_{\lambda \sigma}^{\alpha}\left(\partial_{\mu} \Gamma_{\alpha \nu}^{\sigma}+\frac{2}{3} \Gamma_{\mu \tau}^{\sigma} \Gamma_{\nu \alpha}^{\tau}\right)$,

where $\Lambda= \pm 1 / \ell^{2}$ is the cosmological constant and $\mu$ the Chern-Simons coupling. Here we consider $\Lambda=-1 / \ell^{2}$. Notice that our procedure for computing charges does not depend on the boundary terms needed to held a welldefined variational principle [96-98]. 
Introducing the Einstein tensor

$$
\mathcal{G}_{\mu \nu} \equiv R_{\mu \nu}-\frac{1}{2} g_{\mu \nu} R+\Lambda g_{\mu \nu}
$$

and the Hodge-dualized Cotton tensor

$$
C_{\mu \nu} \equiv \epsilon_{\mu}^{\alpha \beta} \nabla_{\alpha}\left(R_{\beta \nu}-\frac{1}{4} g_{\beta \nu} R\right)
$$

the equations of motion are

$$
\mathcal{G}_{\mu \nu}+\frac{1}{\mu} C_{\mu \nu}=0
$$

Any solution of Einstein gravity with negative cosmological constant is automatically a solution of TMG. In particular, the metric (1) is a solution of (10).

\section{A. Charge algebra}

The conserved charges in TMG are modified with respect to their expressions in Einstein gravity and will be denoted with a tilde. The infinitesimal charge difference between two metrics $\bar{g}$ and $g=\bar{g}+\delta g$ associated to an $\mathrm{AKV} \xi$ is given by

$$
\delta \tilde{Q}_{\xi}[g ; \bar{g}]=\int_{C} \sqrt{-g} \tilde{k}_{\xi}^{\mu \nu}[\delta g ; g] \epsilon_{\mu \nu \alpha} \mathrm{d} x^{\alpha},
$$

where the expression of the 1-form $\tilde{k}_{\xi}^{\mu \nu}$ can be found in [78] (see also Eq. (142) of [71]) and $C$ is a fixed-time contour at the AdS boundary. The Virasoro-Kač-Moody charges then become

$$
\begin{aligned}
& \tilde{\mathcal{L}}_{m}=\frac{1}{2 \pi \mu \ell} \int_{0}^{2 \pi} \mathrm{d} \phi e^{i m x^{+}}\left[(\mu \ell+1) \bar{L}-(\mu \ell-1) \Delta\left(\partial_{+} \bar{P}\right)^{2}\right], \\
& \tilde{\mathcal{P}}_{m}=\frac{1}{2 \pi \mu \ell} \int_{0}^{2 \pi} \mathrm{d} \phi e^{i m x^{+}}(\mu \ell-1)\left(2 \Delta \partial_{+} \bar{P}+\Delta\right)
\end{aligned}
$$

satisfying the same algebra as (4) with modified central extensions given by

$$
\tilde{c}_{R}=\left(1+\frac{1}{\mu \ell}\right) c_{R}, \quad \tilde{k}_{K M}=\left(1-\frac{1}{\mu \ell}\right) k_{K M} .
$$

We will hereafter focus on the signs of $\tilde{c}_{R}$ and $\tilde{k}_{K M}$ pertaining to holographic WCFTs, hence we will consider $\mu \ell \leq-1$ or $\mu \ell \geq 1$.

\section{B. Special points}

We see from (12) and (13) that at the point $\mu \ell=1$, the asymptotic symmetry group reduces to a chiral Virasoro algebra, because the Kač-Moody charges vanish (i.e., become trivial).
To study the point $\mu \ell=-1$, we perform a redefinition of the arbitrary function $\sigma$

$$
\sigma\left(x^{+}\right) \rightarrow \sigma\left(x^{+}\right)+\sigma\left(x^{+}\right) \partial_{+} \bar{P}\left(x^{+}\right) .
$$

Under this shift the charges become

$$
\begin{aligned}
& \tilde{\mathcal{L}}_{m}=\frac{1}{2 \pi}\left(1+\frac{1}{\mu \ell}\right) \int_{0}^{2 \pi} \mathrm{d} \phi e^{i m x^{+}} \bar{L}, \\
& \tilde{\mathcal{P}}_{m}=\frac{1}{2 \pi}\left(1-\frac{1}{\mu \ell}\right) \int_{0}^{2 \pi} \mathrm{d} \phi e^{i m x^{+}}\left(2 \Delta \partial_{+} \bar{P}+\Delta\right)
\end{aligned}
$$

and the mixed commutator $i\left\{\mathcal{L}_{m}, \mathcal{P}_{n}\right\}$ in (4) vanishes. The action of our redefinition has been to disentangle the Virasoro sector of the algebra from the Kač-Moody one, for any value of $\mu$. Now, at $\mu \ell=-1$ the Virasoro generators (15) vanish identically as does the central charge, and the total algebra is simply given by a pure $\hat{u}(1)$ Kač-Moody:

$$
i\left\{\tilde{\mathcal{P}}_{m}, \tilde{\mathcal{P}}_{n}\right\}=k_{K M} m \delta_{m,-n} .
$$

This shows that there exists a particular value of the ChernSimons coupling $(\mu \ell=-1)$ where the total asymptotic algebra of 3-dimensional TMG with negative cosmological constant is a $\hat{u}(1)$ Kač-Moody current algebra.

\section{BLACK HOLES}

The BTZ metric in ADM form is

$$
\mathrm{d} s^{2}=-N^{2} \mathrm{~d} t^{2}+\frac{\mathrm{d} r^{2}}{N^{2}}+r^{2}\left(N^{\phi} \mathrm{d} t+\mathrm{d} \phi\right)^{2},
$$

with

$N^{2}=-8 G M+\frac{r^{2}}{\ell^{2}}+\frac{16 G^{2} J^{2}}{r^{2}}=\frac{\left(r^{2}-r_{+}^{2}\right)\left(r^{2}-r_{-}^{2}\right)}{r^{2} \ell^{2}}$

and

$$
N^{\phi}=-\frac{4 G J}{r^{2}} .
$$

The black hole horizons are located at

$$
r_{ \pm}=\sqrt{2 G \ell(\ell M+J)} \pm \sqrt{2 G \ell(\ell M-J)} .
$$

The black hole mass $\tilde{M}=Q_{\partial_{t}}$ and angular momentum $\tilde{J}=Q_{-\partial_{\phi}}$ in TMG depart from their values $M$ and $J$ in pure gravity and are given by

$$
\begin{gathered}
\ell \tilde{M}=\ell M-\frac{J}{\mu \ell}, \\
\tilde{J}=J-\frac{M}{\mu} .
\end{gathered}
$$


Absence of naked singularities imposes $\ell M \geq|J|$. Therefore, in order to have positive energies $\ell \tilde{M}>0$, we need to consider $|\mu| \geq \frac{1}{\ell}$, which is the condition we had already obtained under (13). At the special points $\mu \ell= \pm 1$ we have the extremality conditions

$$
\ell \tilde{M}=\mp \tilde{J} .
$$

The BTZ entropy in TMG has been computed in [99-102] resulting in

$$
\tilde{S}=\frac{\pi r_{+}}{2 G}-\frac{\pi r_{-}}{2 G \mu \ell} .
$$

We expect this to be reproduced by counting the degeneracy of states in the dual WCFT. In the quadratic ensemble, the warped Cardy formula takes the form [71]

$$
S_{\mathrm{WCFT}}=4 \pi \sqrt{-\tilde{\mathcal{P}}_{0}^{\text {vac }} \tilde{\mathcal{P}}_{0}}+4 \pi \sqrt{-\tilde{\mathcal{L}}_{0}^{\text {vac }} \tilde{\mathcal{L}}_{0}} .
$$

In this expression the subscript vac refers to the charges of the vacuum. Here, the vacuum is global $\mathrm{AdS}_{3}$, whose charges are $M=-1 / 8 G$ and $J=0$. For the BTZ black hole, the zero modes in (15) are given by

$$
\tilde{\mathcal{L}}_{0}=\left(1+\frac{1}{\mu \ell}\right)\left(\frac{\ell M-J}{2}\right),
$$

and

$$
\tilde{\mathcal{P}}_{0}=\left(1-\frac{1}{\mu \ell}\right)\left(\frac{\ell M+J}{2}\right) .
$$

Plugging this in (25) using (20), one finds $\tilde{S}=S_{\mathrm{WCFT}}$ [103]. At the special points $\mu \ell= \pm 1$, one observes that the BTZ black hole entropy is reproduced from the contributions of a chiral Virasoro or $\hat{u}(1)$ Kač-Moody current algebra only.

\section{GRAVITONS}

In this section we solve the spin-2 linearized perturbation around the $\mathrm{AdS}_{3}$ background in TMG. While we begin following closely [33], we then require different conditions on the perturbations-due to the different asymptotic symmetries under consideration. We end up with numerous perturbations solving the linearized TMG equations of motion of which we keep the ones with finite energy and regular at the origin.
We consider $\mathrm{AdS}_{3}$ in global coordinates [104]

$$
\begin{aligned}
\mathrm{d} s^{2} & =-\frac{1}{4} \ell^{2}\left(-4 \mathrm{~d} \rho^{2}+\mathrm{d} x^{+2}+2 \mathrm{~d} x^{+} \mathrm{d} x^{-} \cosh (2 \rho)+\mathrm{d} x^{-2}\right) \\
& :=\bar{g}_{\mu \nu} \mathrm{d} x^{\mu} \mathrm{d} x^{\nu} .
\end{aligned}
$$

The isometry group of the metric (28) is $S L(2, \mathbb{R})_{L} \times$ $S L(2, \mathbb{R})_{R}$ with generators $\bar{L}_{0, \pm 1}$ and $L_{0, \pm 1}$ respectively. We will single out a $U(1) \times S L(2, \mathbb{R})_{R}$ subalgebra compatible with the CSS boundary conditions to classify the perturbations, the $U(1)$ factor being generated by $P_{0}=i \partial_{-}=\bar{L}_{0}$ and the relevant $S L(2, \mathbb{R})_{R}$ generators given by

$$
\begin{gathered}
L_{0}=i \partial_{+}, \\
L_{1}=i e^{i x^{+}}\left[\frac{\cosh 2 \rho}{\sinh 2 \rho} \partial_{+}-\frac{1}{\sinh 2 \rho} \partial_{-}-\frac{i}{2} \partial_{\rho}\right] .
\end{gathered}
$$

We write linearized excitations around the $\mathrm{AdS}_{3}$ background metric $\bar{g}$ as

$$
g_{\mu \nu}=\bar{g}_{\mu \nu}+h_{\mu \nu},
$$

with $h_{\mu \nu}$ a small perturbation. The linearized equations of motion in TMG are

$$
\mathcal{G}_{\mu \nu}^{(1)}+\frac{1}{\mu} C_{\mu \nu}^{(1)}=0,
$$

where explicit expressions for the linearized Einstein and Cotton tensors can be found in $[33,45]$. In transverse and traceless gauge

$$
\bar{\nabla}_{\mu} h^{\mu}{ }_{\nu}=h=0,
$$

the equations of motion can be recast as $[33,45]$

$$
\left(\bar{\nabla}^{2}+\frac{2}{\ell^{2}}\right)\left(h_{\mu \nu}+\frac{1}{\mu} \varepsilon_{\mu}^{\alpha \beta} \bar{\nabla}_{\alpha} h_{\beta \nu}\right)=0 .
$$

We want to use the $U(1) \times S L(2, \mathbb{R})_{R}$ algebra to classify linear perturbations. Consider thus primary states with weight $(h, p)$ :

$$
L_{0}\left|h_{\mu \nu}\right\rangle=h\left|h_{\mu \nu}\right\rangle, \quad P_{0}\left|h_{\mu \nu}\right\rangle=p\left|h_{\mu \nu}\right\rangle,
$$

which implies

$$
h_{\mu \nu}=e^{-i\left(h x^{+}+p x^{-}\right)} F_{\mu \nu}(\rho) .
$$

The transverse, traceless, and highest-weight condition $L_{1}\left|h_{\mu \nu}\right\rangle=\operatorname{Lie}_{L_{1}}\left|h_{\mu \nu}\right\rangle=0$ will strongly constrain the form of $F_{\mu \nu}(\rho)$, whose components will depend on $p, h$, and a set of integration constants. Inserting the result in the equations of motion, and requiring finiteness of the 
linearized energy of the real part of the perturbation (Eq. (69) of [33]), regularity at the origin and to satisfy the CSS boundary conditions singles out 3 solutions:

(i) Massive mode: $h=\frac{1}{2}(3-\mu \ell), p=-\frac{1}{2}(1+\mu \ell)$ and $\beta=0$ hereunder, with energy

$$
E=-\frac{\alpha^{2}(\mu \ell-1)^{2}(\mu \ell+1)}{256 G \mu \ell^{6}(4-2 \mu \ell)}
$$

for $\mu \ell<2$ and diverge for $\mu \ell \geq 2$.

(ii) Right graviton mode: $h=2, p=0$ and $\beta=0$ hereunder, with energy

$$
E=\frac{\alpha^{2}(\mu \ell+1)}{384 G \mu \ell^{6}} .
$$

(iii) Right photon mode: $h=1, p=0$ and $\alpha=0$ hereunder, with energy [105]

$$
E=\frac{\beta^{2}(\mu \ell-1)}{32 G \mu \ell^{6}}
$$

with explicit wave functions

$$
\begin{aligned}
& F_{++}= \frac{1}{4} \cosh ^{4-2 h} \rho \tanh ^{p-h} \rho\left(4 \beta \tanh ^{2} \rho+\alpha \tanh ^{4} \rho\right) \\
& F_{+-}=\frac{1}{2} \cosh ^{2(1-h)} \rho \tanh ^{p-h} \rho\left(\beta \tanh ^{2} \rho\right) \\
& F_{+\rho}= \frac{i}{32} \sinh ^{-1} \rho \cosh ^{-(1+2 h)} \rho \tanh ^{p-h} \rho[4 \cosh 2 \rho(2 \beta-\alpha) \\
&-8 \beta+3 \alpha+\alpha(\cosh 4 \rho)] \\
& F_{--}=0 \\
& F_{-\rho}=-\frac{i}{4} \cosh ^{-1} \rho \sinh ^{-1} \rho \sinh ^{-h} 2 \rho \tanh { }^{p-h} \rho \\
& \quad \times\left[\sinh ^{h} 2 \rho \cosh ^{-2 h} \rho\left((-\beta) \cosh ^{2} \rho+\beta\right)\right] \\
& F_{\rho \rho}= \sinh ^{-h-2} 2 \rho \tanh ^{p-h} \rho\left[\cosh ^{4-2 h} \rho \sinh ^{h} 2 \rho\right. \\
&\left.\times\left((4 \beta-\alpha) \tanh ^{4} \rho\right)\right]
\end{aligned}
$$

The first two modes were present in [33], and their energies coincide with (70)-(71) of that Ref. [106]. There is no Left graviton mode with $h=0$ and $p=2$, as it is excluded by the CSS boundary conditions. Instead, there is a new solution, the Right photon mode which satisfies CSS but not Brown-Henneaux.

For $\mu \ell<-1$, the massive mode has a negative energy while the graviton and the photon mode have a positive energy. At the special point $\mu \ell=-1$ the massive mode and the graviton have a zero energy while the photon has a positive energy. At the special point $\mu \ell=1$ the massive mode and the photon have zero energy while the graviton has a positive energy. For $\mu \ell>1$ the massive mode has a negative energy while the graviton and the photon mode both have a positive energy. We thus see that the two special points $\mu \ell= \pm 1$ allow us to avoid negative energy, while the right moving graviton or photon carry no energy.

\section{SUMMARY AND FURTHER DEVELOPMENTS}

In this work we investigated CSS boundary conditions in TMG for $|\mu| \ell \geq 1$ (for which BTZ black holes have a positive energy) and noticed two special points in the space of couplings.

At $\mu \ell=1$ :

(i) The $\hat{u}(1)$ Kač-Moody charges and level vanish;

(ii) BTZ black holes have positive energy, an entropy reproduced by a chiral half of the Cardy formula and $\ell \tilde{M}=-\tilde{J}$;

(iii) The massive graviton and boundary photon acquire vanishing energy, while the boundary graviton has positive energy.

At $\mu \ell=-1$ :

(i) The Virasoro generators and central charge vanish;

(ii) BTZ black holes have positive energy, an entropy reproduced by the $\hat{u}(1)$ contribution of the warped Cardy formula and $\ell \tilde{M}=\tilde{J}$;

(iii) The massive and boundary gravitons acquire vanishing energy, while the boundary photon has positive energy.

This suggests that TMG with CSS boundary conditions at these two points might be a stable and consistent gravity theory dual either to a holomorphic CFT, or a theory with $\hat{u}(1)$ affine symmetry.

In this work we have considered a phase space consisting of Einstein solutions. It is known however that there exist a large variety of solutions to TMG which are not Einstein [107]. CSS should therefore be generalized to TMG, in the spirit of [49]. In particular, logarithmic solutions are likely to arise at the special points. This might in turn lead to the definition of "logarithmic WCFT" that could be dual to a relaxation of CSS in TMG at the special points. A positive energy theorem should also be proven. A preliminary step would be to show that the only stationary, axially symmetric solutions of the theory are BTZ black holes. This is true for chiral gravity [45], even though non-Einstein timedependent solutions do exist [108]. It would also be interesting to evaluate the Euclidean sum over geometries and determine what type of partition it can be identified with.

\section{ACKNOWLEDGMENTS}

We thank Alessio Caddeo, Marc Henneaux, Blagoje Oblak, Antonin Rovai, Wei Song and Bayram Tekin for 
valuable discussions. The work of L. C. is supported by the ERC Advanced Grant "High-Spin-Grav". S. D. is a Research Associate of the Fonds de la Recherche Scientifique F. R. S.-FNRS (Belgium). S. D. was supported in part by IISN-Belgium (convention 4.4503.15) and benefited from the support of the Solvay Family. S. D. acknowledges support of the Fonds de la Recherche Scientifique F. R.S.-FNRS (Belgium) through the CDR project C 60/5-CDR/OL "Horizon holography: black holes and field theories" (2020-2022).
[1] J. Bekenstein, Black holes and the second law, Lett. Nuovo Cimento 4, 737 (1972).

[2] J. D. Bekenstein, Black holes and entropy, Phys. Rev. D 7, 2333 (1973).

[3] S. Hawking, Black hole explosions, Nature (London) 248, 30 (1974).

[4] S. Hawking, Particle creation by black holes, Commun. Math. Phys. 43, 199 (1975); Erratum, Commun. Math. Phys. 46, 206 (1976).

[5] J. R. Gott and M. Alpert, General relativity in a $(2+1)$ dimensional space-time, Gen. Relativ. Gravit. 16, 243 (1984).

[6] S. Giddings, J. Abbott, and K. Kuchar, Einstein's theory in a three-dimensional space-time, Gen. Relativ. Gravit. 16, 751 (1984).

[7] S. Deser, R. Jackiw, and G. 't Hooft, Three-dimensional Einstein gravity: Dynamics of flat space, Ann. Phys. (N.Y.) 152, 220 (1984).

[8] J. D. Barrow, A. B. Burd, and D. Lancaster, Threedimensional classical spacetimes, Classical Quantum Gravity 3, 551 (1986).

[9] M. Banados, C. Teitelboim, and J. Zanelli, The Black Hole in Three-Dimensional Space-Time, Phys. Rev. Lett. 69, 1849 (1992).

[10] C. Bunster, M. Henneaux, A. Perez, D. Tempo, and R. Troncoso, Generalized black holes in three-dimensional spacetime, J. High Energy Phys. 05 (2014) 031.

[11] J. Brown and M. Henneaux, Central charges in the canonical realization of asymptotic symmetries: An example from three-dimensional gravity, Commun. Math. Phys. 104, 207 (1986).

[12] J. M. Maldacena, The large N limit of superconformal field theories and supergravity, Int. J. Theor. Phys. 38, 1113 (1999).

[13] A. Strominger, Black hole entropy from near horizon microstates, J. High Energy Phys. 02 (1998) 009.

[14] G. Penington, Entanglement wedge reconstruction and the information paradox, J. High Energy Phys. 09 (2020) 002.

[15] A. Almheiri, N. Engelhardt, D. Marolf, and H. Maxfield, The entropy of bulk quantum fields and the entanglement wedge of an evaporating black hole, J. High Energy Phys. 12 (2019) 063.

[16] A. Almheiri, T. Hartman, J. Maldacena, E. Shaghoulian, and A. Tajdini, Replica wormholes and the entropy of Hawking radiation, J. High Energy Phys. 05 (2020) 013.

[17] C. Teitelboim, Gravitation and Hamiltonian structure in two space-time dimensions, Phys. Lett. 126B, 41 (1983).
[18] R. Jackiw, Lower dimensional gravity, Nucl. Phys. B252, 343 (1985).

[19] A. Almheiri and J. Polchinski, Models of $\mathrm{AdS}_{2}$ backreaction and holography, J. High Energy Phys. 11 (2015) 014.

[20] T. G. Mertens, The Schwarzian theory-Origins, J. High Energy Phys. 05 (2018) 036.

[21] J. Cotler and K. Jensen, A theory of reparameterizations for $\mathrm{AdS}_{3}$ gravity, J. High Energy Phys. 02 (2019) 079.

[22] J. L. Cardy, Operator content of two-dimensional conformally invariant theories, Nucl. Phys. B270, 186 (1986).

[23] T. Hartman, C. A. Keller, and B. Stoica, Universal spectrum of $2 \mathrm{~d}$ conformal field theory in the large c limit, J. High Energy Phys. 09 (2014) 118.

[24] E. Witten, Three-dimensional gravity revisited, arXiv: 0706.3359.

[25] A. Maloney and E. Witten, Quantum gravity partition functions in three dimensions, J. High Energy Phys. 02 (2010) 029.

[26] M. R. Gaberdiel, Constraints on extremal self-dual CFTs, J. High Energy Phys. 11 (2007) 087.

[27] M. R. Gaberdiel and C. A. Keller, Modular differential equations and null vectors, J. High Energy Phys. 09 (2008) 079.

[28] M. R. Gaberdiel, C. A. Keller, and R. Volpato, Genus two partition functions of chiral conformal field theories, Commun. Numer. Theor. Phys. 4, 295 (2010).

[29] C. A. Keller and A. Maloney, Poincare series, 3D gravity and CFT spectroscopy, J. High Energy Phys. 02 (2015) 080 .

[30] N. Benjamin, H. Ooguri, S.-H. Shao, and Y. Wang, Lightcone modular bootstrap and pure gravity, Phys. Rev. D 100, 066029 (2019).

[31] L. F. Alday and J.-B. Bae, Rademacher expansions and the spectrum of 2d CFT, arXiv:2001.00022.

[32] N. Benjamin, S. Collier, and A. Maloney, Pure gravity and conical defects, J. High Energy Phys. 09 (2020) 034.

[33] W. Li, W. Song, and A. Strominger, Chiral gravity in three dimensions, J. High Energy Phys. 04 (2008) 082.

[34] S. Deser, R. Jackiw, and S. Templeton, Topologically massive gauge theories, Ann. Phys. (N.Y.) 140, 372 (1982); Erratum, Ann. Phys. (N.Y.) 185, 406 (1988).

[35] S. Deser, R. Jackiw, and S. Templeton, Three-Dimensional Massive Gauge Theories, Phys. Rev. Lett. 48, 975 (1982).

[36] S. Carlip, S. Deser, A. Waldron, and D. Wise, Cosmological topologically massive gravitons and photons, Classical Quantum Gravity 26, 075008 (2009). 
[37] D. Grumiller and N. Johansson, Instability in cosmological topologically massive gravity at the chiral point, J. High Energy Phys. 07 (2008) 134.

[38] W. Li, W. Song, and A. Strominger, Comment on 'Cosmological topological massive gravitons and photons', arXiv:0805.3101.

[39] M.-i. Park, Constraint dynamics and gravitons in three dimensions, J. High Energy Phys. 09 (2008) 084.

[40] D. Grumiller, R. Jackiw, and N. Johansson, Canonical analysis of cosmological topologically massive gravity at the chiral point, arXiv:0806.4185.

[41] S. Carlip, S. Deser, A. Waldron, and D. Wise, Topologically massive AdS gravity, Phys. Lett. B 666, 272 (2008).

[42] S. Carlip, The constraint algebra of topologically massive AdS gravity, J. High Energy Phys. 10 (2008) 078.

[43] G. Giribet, M. Kleban, and M. Porrati, Topologically massive gravity at the chiral point is not chiral, J. High Energy Phys. 10 (2008) 045.

[44] A. Strominger, A simple proof of the chiral gravity conjecture, arXiv:0808.0506.

[45] A. Maloney, W. Song, and A. Strominger, Chiral gravity, log gravity and extremal CFT, Phys. Rev. D 81, 064007 (2010).

[46] S. Carlip, Chiral topologically massive gravity and extremal B-F scalars, J. High Energy Phys. 09 (2009) 083.

[47] K. Skenderis, M. Taylor, and B. C. van Rees, Topologically massive gravity and the AdS/CFT correspondence, J. High Energy Phys. 09 (2009) 045.

[48] T. Andrade and D. Marolf, No chiral truncation of quantum log gravity?, J. High Energy Phys. 03 (2010) 029.

[49] M. Henneaux, C. Martinez, and R. Troncoso, More on asymptotically anti-de Sitter spaces in topologically massive gravity, Phys. Rev. D 82, 064038 (2010).

[50] N. Lashkari and A. Maloney, Topologically massive gravity and Ricci-Cotton flow, Classical Quantum Gravity 28, 105007 (2011).

[51] C. Cunliff, Topologically massive gravity from the outside In, Classical Quantum Gravity 28, 195024 (2011).

[52] C. Nazaroglu, Y. Nutku, and B. Tekin, Covariant symplectic structure and conserved charges of topologically massive gravity, Phys. Rev. D 83, 124039 (2011).

[53] A. Castro, N. Lashkari, and A. Maloney, Quantum topologically massive gravity in de Sitter space, J. High Energy Phys. 08 (2011) 040.

[54] S. Dengiz, E. Kilicarslan, and B. Tekin, Scattering in topologically massive gravity, chiral gravity and the corresponding Anyon-Anyon potential energy, Phys. Rev. D 89, 024033 (2014).

[55] E. Altas and B. Tekin, Linearization instability of chiral gravity, Phys. Rev. D 97, 124068 (2018).

[56] D. Grumiller and I. Sachs, AdS (3)/LCFT (2) $\rightarrow>$ Correlators in cosmological topologically massive gravity, J. High Energy Phys. 03 (2010) 012.

[57] M. R. Gaberdiel, D. Grumiller, and D. Vassilevich, Graviton 1-loop partition function for 3-dimensional massive gravity, J. High Energy Phys. 11 (2010) 094.

[58] D. Grumiller, N. Johansson, and T. Zojer, Short-cut to new anomalies in gravity duals to logarithmic conformal field theories, J. High Energy Phys. 01 (2011) 090.
[59] D. Grumiller, W. Riedler, J. Rosseel, and T. Zojer, Holographic applications of logarithmic conformal field theories, J. Phys. A 46, 494002 (2013).

[60] Y. Mvondo-She and K. Zoubos, On the combinatorics of partition functions in $\mathrm{AdS}_{3} / \mathrm{LCFT}_{2}$, J. High Energy Phys. 05 (2019) 097.

[61] G. Compre, W. Song, and A. Strominger, New boundary conditions for AdS3, J. High Energy Phys. 05 (2013) 152.

[62] C. Troessaert, Enhanced asymptotic symmetry algebra of $\mathrm{AdS}_{3}$, J. High Energy Phys. 08 (2013) 044.

[63] S. G. Avery, R. R. Poojary, and N. V. Suryanarayana, An $\mathrm{sl}(2, \mathbb{R})$ current algebra from $\mathrm{AdS}_{3}$ gravity, J. High Energy Phys. 01 (2014) 144.

[64] C. Troessaert, Poisson structure of the boundary gravitons in 3D gravity with negative $\Lambda$, Classical Quantum Gravity 32, 235019 (2015).

[65] L. Donnay, G. Giribet, H. A. Gonzalez, and M. Pino, Supertranslations and Superrotations at the Black Hole Horizon, Phys. Rev. Lett. 116, 091101 (2016).

[66] A. Prez, D. Tempo, and R. Troncoso, Boundary conditions for general relativity on $\mathrm{AdS}_{3}$ and the $\mathrm{KdV}$ hierarchy, J. High Energy Phys. 06 (2016) 103.

[67] H. Afshar, D. Grumiller, and M. Sheikh-Jabbari, Near horizon soft hair as microstates of three dimensional black holes, Phys. Rev. D 96, 084032 (2017).

[68] H. Afshar, D. Grumiller, W. Merbis, A. Perez, D. Tempo, and R. Troncoso, Soft hairy horizons in three spacetime dimensions, Phys. Rev. D 95, 106005 (2017).

[69] D. Grumiller, W. Merbis, and M. Riegler, Most general flat space boundary conditions in three-dimensional Einstein gravity, Classical Quantum Gravity 34, 184001 (2017).

[70] D. M. Hofman and A. Strominger, Chiral Scale and Conformal Invariance in 2D Quantum Field Theory, Phys. Rev. Lett. 107, 161601 (2011).

[71] S. Detournay, T. Hartman, and D. M. Hofman, Warped conformal field theory, Phys. Rev. D 86, 124018 (2012).

[72] J. M. Bardeen and G. T. Horowitz, The extreme Kerr throat geometry: A vacuum analog of $\operatorname{AdS}(2)$ x S**2, Phys. Rev. D 60, 104030 (1999).

[73] O. J. Dias, R. Emparan, and A. Maccarrone, Microscopic theory of black hole superradiance, Phys. Rev. D 77, 064018 (2008).

[74] M. Guica, T. Hartman, W. Song, and A. Strominger, The Kerr/CFT correspondence, Phys. Rev. D 80, 124008 (2009).

[75] M. Banados, G. Barnich, G. Compere, and A. Gomberoff, Three dimensional origin of Godel spacetimes and black holes, Phys. Rev. D 73, 044006 (2006).

[76] D. Anninos, W. Li, M. Padi, W. Song, and A. Strominger, Warped AdS(3) black holes, J. High Energy Phys. 03 (2009) 130.

[77] G. Compere and S. Detournay, Centrally extended symmetry algebra of asymptotically Godel spacetimes, J. High Energy Phys. 03 (2007) 098.

[78] G. Compere and S. Detournay, Semi-classical central charge in topologically massive gravity, Classical Quantum Gravity 26, 012001 (2009).

[79] G. Compere and S. Detournay, Boundary conditions for spacelike and timelike warped AdS_3 spaces in 
topologically massive gravity, J. High Energy Phys. 08 (2009) 092.

[80] M. Blagojevic and B. Cvetkovic, Asymptotic structure of topologically massive gravity in spacelike stretched AdS sector, J. High Energy Phys. 09 (2009) 006.

[81] M. Henneaux, C. Martinez, and R. Troncoso, Asymptotically warped anti-de Sitter spacetimes in topologically massive gravity, Phys. Rev. D 84, 124016 (2011).

[82] D. M. Hofman and B. Rollier, Warped conformal field theory as lower spin gravity, Nucl. Phys. B897, 1 (2015).

[83] A. Castro, D. M. Hofman, and G. Srosi, Warped Weyl fermion partition functions, J. High Energy Phys. 11 (2015) 129.

[84] A. Castro, D. M. Hofman, and N. Iqbal, Entanglement entropy in warped conformal field theories, J. High Energy Phys. 02 (2016) 033.

[85] W. Song, Q. Wen, and J. Xu, Generalized Gravitational Entropy for Warped Anti-de Sitter Space, Phys. Rev. Lett. 117, 011602 (2016).

[86] W. Song, Q. Wen, and J. Xu, Modifications to holographic entanglement entropy in warped CFT, J. High Energy Phys. 02 (2017) 067.

[87] W. Song and J. Xu, Correlation functions of warped CFT, J. High Energy Phys. 04 (2018) 067.

[88] A. Castro, C. Keeler, and P. Szepietowski, Tweaking one-loop determinants in $\mathrm{AdS}_{3}$, J. High Energy Phys. 10 (2017) 070.

[89] K. Jensen, Locality and anomalies in warped conformal field theory, J. High Energy Phys. 12 (2017) 111.

[90] T. Azeyanagi, S. Detournay, and M. Riegler, Warped black holes in lower-spin gravity, Phys. Rev. D 99, 026013 (2019).

[91] A. Aggarwal, A. Castro, and S. Detournay, Warped symmetries of the Kerr black hole, J. High Energy Phys. 01 (2020) 016.

[92] L. Apolo, H. Jiang, W. Song, and Y. Zhong, Swing surfaces and holographic entanglement beyond AdS/CFT, arXiv: 2006.10740.

[93] L. Apolo, H. Jiang, W. Song, and Y. Zhong, Modular Hamiltonians in flat holography and (W)AdS/WCFT, J. High Energy Phys. 09 (2020) 033.

[94] L. Apolo and W. Song, Bootstrapping holographic warped CFTs or: how I learned to stop worrying and tolerate negative norms, J. High Energy Phys. 07 (2018) 112.
[95] P. Chaturvedi, Y. Gu, W. Song, and B. Yu, A note on the complex SYK model and warped CFTs, J. High Energy Phys. 12 (2018) 101.

[96] G. Barnich and F. Brandt, Covariant theory of asymptotic symmetries, conservation laws and central charges, Nucl. Phys. B633, 3 (2002).

[97] G. Barnich and G. Compere, Surface charge algebra in gauge theories and thermodynamic integrability, J. Math. Phys. (N.Y.) 49, 042901 (2008).

[98] G. Compère and A. Fiorucci, Advanced Lectures on General Relativity (Springer, Cham, 2019).

[99] P. Kraus and F. Larsen, Holographic gravitational anomalies, J. High Energy Phys. 01 (2006) 022.

[100] S. N. Solodukhin, Holography with gravitational ChernSimons, Phys. Rev. D 74, 024015 (2006).

[101] Y. Tachikawa, Black hole entropy in the presence of Chern-Simons terms, Classical Quantum Gravity 24, 737 (2007).

[102] A. Bouchareb and G. Clement, Black hole mass and angular momentum in topologically massive gravity, Classical Quantum Gravity 24, 5581 (2007).

[103] This had been shown for any diffeomorphism-invariant higher curvature theory in [109].

[104] Abusing notation, we call again the holographic coordinate $\rho$, although it is the logarithm of the Fefferman-Graham coordinate (also spelled $\rho$ ). The latter has dimension length while the former is dimensionless.

[105] Using the same terminology as [78], we call the photon the mode with $h=1$.

[106] Remember that left and right are flipped and that $\mu_{\text {here }}=-\mu_{\text {there }}$ because of a different convention in the $\varepsilon$ symbol. Our convention is $\varepsilon_{+-\rho}=-1$.

[107] D. D. Chow, C. Pope, and E. Sezgin, Classification of solutions in topologically massive gravity, Classical Quantum Gravity 27, 105001 (2010).

[108] G. Compere, S. de Buyl, and S. Detournay, Non-Einstein geometries in chiral gravity, J. High Energy Phys. 10 (2010) 042.

[109] C. Zwikel, BTZ black holes and flat space cosmologies in higher derivative theories, Classical Quantum Gravity 34, 085003 (2017). 